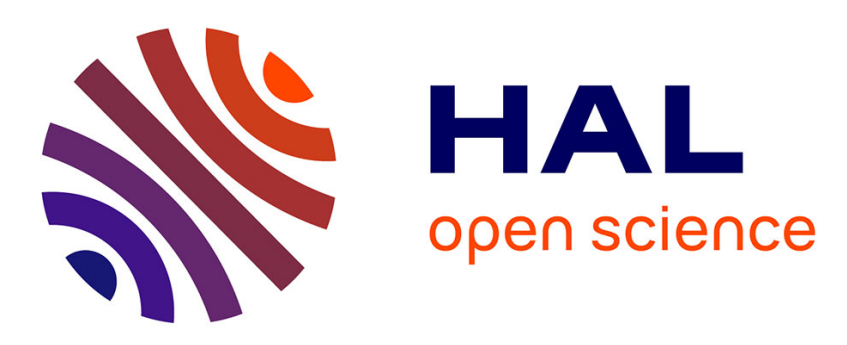

\title{
Capture of solar wind alpha-particles by the Martian atmosphere
}

\author{
Gérard Chanteur, E. Dubinin, Ronan Modolo, M. Fraenz
}

\section{To cite this version:}

Gérard Chanteur, E. Dubinin, Ronan Modolo, M. Fraenz. Capture of solar wind alphaparticles by the Martian atmosphere. Geophysical Research Letters, 2009, 36 (23), pp.L23105. 10.1029/2009GL040235. hal-00429684

\section{HAL Id: hal-00429684 \\ https://hal.science/hal-00429684}

Submitted on 28 Feb 2016

HAL is a multi-disciplinary open access archive for the deposit and dissemination of scientific research documents, whether they are published or not. The documents may come from teaching and research institutions in France or abroad, or from public or private research centers.
L'archive ouverte pluridisciplinaire HAL, est destinée au dépôt et à la diffusion de documents scientifiques de niveau recherche, publiés ou non, émanant des établissements d'enseignement et de recherche français ou étrangers, des laboratoires publics ou privés. 


\title{
Capture of solar wind alpha-particles by the Martian atmosphere
}

\author{
G. M. Chanteur, ${ }^{1}$ E. Dubinin, ${ }^{2}$ R. Modolo, ${ }^{3,4}$ and M. Fraenz ${ }^{2}$ \\ Received 24 July 2009; revised 23 October 2009; accepted 30 October 2009; published 9 December 2009.
}

[1] Integration along $\mathrm{He}^{++}$test-particle trajectories in the self-consistent electromagnetic fields generated by threedimensional hybrid simulations of the solar wind/Mars interaction is used to evaluate the removal of solar wind $\alpha$-particles due to charge-exchange processes with neutral species of the Martian exosphere. The total removal rate of solar wind $\mathrm{He}^{++}$ions, transformed into either singly ionised or neutral helium, is equal to $6.7 \times 10^{23} \mathrm{~s}^{-1}$, which corresponds approximately to $30 \%$ of the flux of solar $\alpha$-particles through the planetary cross-section. The deposition rate of helium neutral atoms, created by double electronic capture on exospheric oxygen, impacting the exobase, and penetrating below where it can be trapped, is about $1.5 \times 10^{23} \mathrm{~s}^{-1}$. That means an important contribution of the solar wind source to the helium balance of the Martian atmosphere. The implantation of the solar helium into the Martian atmosphere shows an asymmetry related to the orientation of the motional electric field of the solar wind, $-\boldsymbol{V}_{S W} \times \boldsymbol{B}_{I M F}$. Citation: Chanteur, G. M., E. Dubinin, R. Modolo, and M. Fraenz (2009), Capture of solar wind alphaparticles by the Martian atmosphere, Geophys. Res. Lett., 36, L23105, doi:10.1029/2009GL040235.

\section{Introduction}

[2] Helium in the atmospheres of the terrestrial planets is generally produced by the surface outgassing of the helium atoms produced by the radioactive decay of uranium $(U)$ and thorium $(T h)$ in the planetary interiors. The first remote measurements of $\mathrm{He}$ at Mars were made on the EUVE satellite orbiting the Earth [Krasnopolsky et al., 1994]. Using He-584A airglow observations Krasnopolsky and Gladstone [1996] concluded that the helium mixing ratio is equal to $4 \pm 2 \mathrm{ppm}$ in the lower atmosphere and the escape rate is $(7.2 \pm 3.6) \times 10^{23} \mathrm{~s}^{-1}$. The first in-situ measurements of ionized planetary helium picked up by the solar wind were carried out onboard the Phobos-2 spacecraft [Barabash and Norberg, 1994; Barabash et al., 1995]. The reported escape fluxes of $\mathrm{He}^{+}$ions equal to $(1.2 \pm 0.6) \times$ $10^{24} \mathrm{~s}^{-1}$ are consistent with the estimations made by Krasnopolsky and Gladstone [1996] taking into account the difference in solar activity. Such high values of the non thermal losses imply that helium formed by the radioactive decay of $U$ and $T h$ could be fully lost in less than one

\footnotetext{
${ }^{1}$ Laboratoire de Physique des Plasmas, Ecole Polytechnique, UPMC, CNRS, Palaiseau, France.

${ }^{2}$ MPS Institut, Katlenburg-Lindau, Germany.

${ }^{3}$ Centre d'étude des environnements terrestre et planétaires, Université de Versailles, Saint-Quentin-en-Yvelines, France.

${ }^{4}$ Laboratoire Atmosphères, Milieux, Observations Spatiales, IPSL, CNRS, Vélizy, France.
}

Copyright 2009 by the American Geophysical Union. 0094-8276/09/2009GL040235 million years: a lifetime of helium on Mars is 0.13 million years [Krasnopolsky and Gladstone, 2005], much shorter than the age of the latest volcanism which is estimated at a few million years [Neukum et al., 2004]. Therefore outgassing of radiogenic helium may be a minor source of helium on Mars. Krasnopolsky and Gladstone [1996] have suggested that $\sim 70 \%$ of the required source flux is supplied by the capture of solar wind $\alpha$-particles with efficiency $\sim 0.3 \pm 0.1$. Recent longer-exposure EUVE observations of

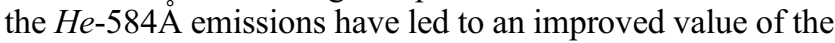
mixing ratio equal to ( $10 \pm 6 \mathrm{ppm})$ although the estimate of the total loss of helium $\left(8 \times 10^{23} \mathrm{~s}^{-1}\right)$ is almost unchanged [Krasnopolsky and Gladstone, 2005]. Krasnopolsky and Gladstone [2005] have concluded that the capture of solar wind $\mathrm{He}^{++}$ions can be the only substantial source of helium on Mars. However, the efficiency of this process has not yet been tested experimentally or accurately tested by global simulations of the solar wind/Mars interaction. The deposition of solar wind matter on Mars was first evaluated by Brecht [1997] by means of mono-species threedimensional hybrid simulations. In these early simulations, the IMF and solar wind protons interacted with a bare and conducting sphere without atmosphere. The deposition of solar wind protons on the surface was of 3-30\% depending upon the ram pressure. A simple scaling to alpha-particles yields the deposition rate of $1.3 \times 10^{6} \mathrm{~cm}^{-2} \mathrm{~s}^{-1}$. However, the nature of the Martian obstacle to the solar wind is more complicated. The solar wind interacts directly with the planetary upper atmosphere and ionosphere inducing a magnetosphere by piling-up and draping the interplanetary magnetic field around the obstacle. This induced magnetosphere protects the low altitude ionosphere and atmosphere from a direct exposure to the solar wind. It is also worth noting that due to their different gyroradii $\alpha$-particles and protons have different dynamics in the Martian environment, resulting in different deposition rates which might not be related by a simple scaling.

[3] Recently, Modolo et al. [2005] designed a more sophisticated 3-D hybrid model including the dynamics of both solar wind $\left(\mathrm{H}^{+}, \mathrm{He}^{++}\right)$and planetary ion species $\left(\mathrm{H}^{+}\right.$, $\mathrm{O}^{+}, \mathrm{O}_{2}^{+}$). In this approach, the Martian neutral environment above the exobase is modeled by spherically symmetric coronas of atomic hydrogen and oxygen, the height profiles of which depend upon the solar EUV flux. The in situ exospheric source of planetary ions is modeled through a self-consistent computation of the local production rate of each planetary species, $\mathrm{H}^{+}$and $\mathrm{O}^{+}$, for each ionization process (photoionization, electron impact, and chargeexchange) making use of charge exchange cross sections, ionization frequencies, given neutral densities, and computed plasma parameters (for details see Modolo et al. [2005]). The ionospheric reservoir is modeled by imposing the upward fluxes of the atomic and molecular oxygen ions at the altitude of the exobase $(250 \mathrm{~km})$. The spatial 
Table 1. Charge Exchange Reactions of $\mathrm{He}^{++}$with Martian Neutrals

\begin{tabular}{ccc}
\hline Channel $^{\mathrm{a}}$ & Charge Exchange Reaction & $\sigma\left(\mathrm{cm}^{-1}\right)$ \\
\hline$(1)^{\mathrm{b}}$ & $H e^{++}+H \rightarrow H e^{+}+H^{+}$ & $3 \times 10^{-16}$ \\
$(2)$ & $H e^{++}+\mathrm{H}_{2} \rightarrow \mathrm{He}^{+}+H_{2}^{+}$ & $2 \times 10^{-16}$ \\
$(3)$ & $\mathrm{He}+\mathrm{He} \rightarrow \mathrm{He}^{++}+\mathrm{He}^{+}$ & $4 \times 10^{-17}$ \\
$(4)^{\mathrm{b}}$ & $\mathrm{He}+\mathrm{O} \rightarrow \mathrm{He}^{++}+\mathrm{O}^{+}$ & $4 \times 10^{-16}$ \\
$(5)$ & $\mathrm{He}+\mathrm{H}_{2} \rightarrow \mathrm{He}+\mathrm{H}^{++}$ & $5 \times 10^{-17}$ \\
$(6)$ & $H e^{++}+\mathrm{He} \rightarrow \mathrm{He}+\mathrm{He}^{++}$ & $2.5 \times 10^{-16}$ \\
$(7)^{\mathrm{b}}$ & $\mathrm{He}+\mathrm{O} \rightarrow \mathrm{He}+\mathrm{O}^{++}$ & $2 \times 10^{-16}$ \\
\hline
\end{tabular}

${ }^{\mathrm{a}}$ Cross sections have been measured or compiled by: (1), Barnett [1990]; (2), Kusakabe et al. [1990]; (3), Kimura [1988]; (4), Barnett [1990]; (5), Kusakabe et al. [1990] and Panov et al. [2002]; (6), Kimura [1988]; and (7), Barnett [1990].

${ }^{\mathrm{b}}$ Most important processes.

resolution of these runs is equal to $300 \mathrm{~km}$. In this paper we estimate the removal rate of solar wind $\mathrm{He}^{++}$ions due to charge-exchange processes on the Martian atmosphere.

\section{Hybrid and Test Particle Simulations}

[4] The solar wind consisting of protons with number density $n\left(H^{+}\right)=2.3 \mathrm{~cm}^{-3}$ and $H e^{++}$ions with number density $n\left(H e^{++}\right) / n\left(H^{+}\right)=0.05$ flows with velocity $V_{s w}=$ $400 \mathrm{~km} \mathrm{~s}^{-1}$ carrying the interplanetary magnetic field $\left(B_{I M F}=\right.$ $3 \mathrm{nT}$ ). Although the hybrid simulations include selfconsistently the dynamics of alpha-particles, the chargeexchange reactions involving $\mathrm{He}^{++}$ions were not taken into account in the direct and consistent simulation because of their negligible effect on the global dynamics. In order to evaluate the removal of the solar wind $\mathrm{He}^{++}$ions due to charge-exchange processes in the Martian atmosphere we need the positions and velocities of these particles with respect to the neutral species bound to the planet. In a stationary state of the Martian environment this information can be reconstructed by computing the motion of $\mathrm{He}^{++}$test particles in the stationary and self-consistent electromagnetic field which is a stored output of the consistent threedimensional hybrid simulation. While particle densities in the self-consistent hybrid simulation are computed as temporal snapshots from the instantaneous positions of the macroparticles, the same technique applied during a run of test particles would require an extremely large number of test macroparticles to reproduce the densities computed as snapshots during the consistent simulation. The computational effort is drastically reduced by adopting the methodology of test particle Monte-Carlo simulations [Haviland, 1965]. According to Haviland [1965] the density in a given volume of phase space is proportional to the residence time of the test particles in this volume. Indeed we do not compute exactly the residence time of the test particles in a given cell, but as the trajectory of each test particle is naturally sampled by the computational time step we just sum, at each time step, the contributions of each test particle to the simulation cells through which the particle is moving: the resulting number of counts in a given volume is thus proportional to the residence time of the test particle in that volume. The time step used for computing the motion of the test particles is small enough, and the number of test particles large enough, to sample correctly the computational domain. For a given number of injected test particles, $10^{5}$ in the present case, their common initial statistical weight is chosen in order to match the physical incoming flux of solar wind alphas. Each test particle is followed from its entry point, chosen at random on the entry face of the solar wind into the computational domain, up to its crossing of the exit face of the simulation box, or up to its final absorption in the dense atmosphere when it crosses the exobase: the weight of the test macroparticle is decreased by the charge exchange collisions as the test particle moves through the neutral background.

[5] The densities of $\mathrm{He}^{++}$ions, computed as snapshots during the self-consistent hybrid simulation, and by integrating along the trajectories of test particles, are in good agreement despite slight differences due to the suppression of the self-consistent temporal fluctuations when using the frozen field with the test particles. The test particle method using the frozen stationary self-consistent field reproduces very well the positions of the bow shock and the magnetic pileup boundary (MPB) fitted from the MGS observations [Vignes et al., 2000]. Both schemes give results enough similar to justify the used test-particle approach. The MPB, characterized by a sharp jump in the magnetic field strength and an increase in the magnetic field line draping [Acuña et al., 1998; Bertucci et al., 2003], is the boundary of the induced magnetosphere formed at Mars [Dubinin et al., 2006].

[6] Alpha particles can be removed from the solar wind by charge exchange reactions with the main Martian neutrals above the exobase: atomic and molecular hydrogen, helium, and atomic oxygen. Table 1 gives the reactions together with their cross sections at $1 \mathrm{keV} / \mathrm{amu}$ and the references of the measurements: channels (1) to (4) produce $\mathrm{He}^{+}$through single electron capture, and channels (5) to (7) produce $\mathrm{He}$ atoms through double electron capture. We consider neither molecular nitrogen, nor carbon monoxyde or dioxyde as they are important only below $200 \mathrm{~km}$ altitude. Let us notice that channel (6) influences neither the balance of solar wind alpha particles, nor the balance of $\mathrm{He}$ atoms in the atmosphere, and thus is not considered. In order to estimate the most important channels we have computed the local reaction rates at $300 \mathrm{~km}$ by making use of the density model of Krasnopolsky [2002, hereafter K2002], and at $2000 \mathrm{~km}$ using Krasnopolsky and Gladstone [1996, hereafter KG1996], corrected by the ratios of densities at $300 \mathrm{~km}$ between Krasnopolsky [2002] and Krasnopolsky and Gladstone [1996]. For atomic oxygen at $300 \mathrm{~km}$ and $2000 \mathrm{~km}$ altitude we have taken into account both the cold and hot coronas according to Kim et al. [1998].

[7] The most important processes, as noted in Table 1, are the charge-exchange reactions with the neutral atoms of oxygen and hydrogen which produce neutral and singly ionized helium. Concerning the local removal rate of solar wind alphas during solar minimum conditions, the case investigated in this paper, these three channels account for $94 \%$ of the total at $300 \mathrm{~km}$ and for $93 \%$ at $2000 \mathrm{~km}$. During solar maximum, these percentages decrease to $85 \%$ and $70 \%$ respectively: missing contributions come mainly from the helium and molecular hydrogen coronas, the latter contributing only at high altitude. During solar minimum conditions, $96 \%$ of the local production rate of $\mathrm{He}$ atoms by double electron capture at low altitude is explained by the sole channel (7), i.e., the contribution from the atomic 

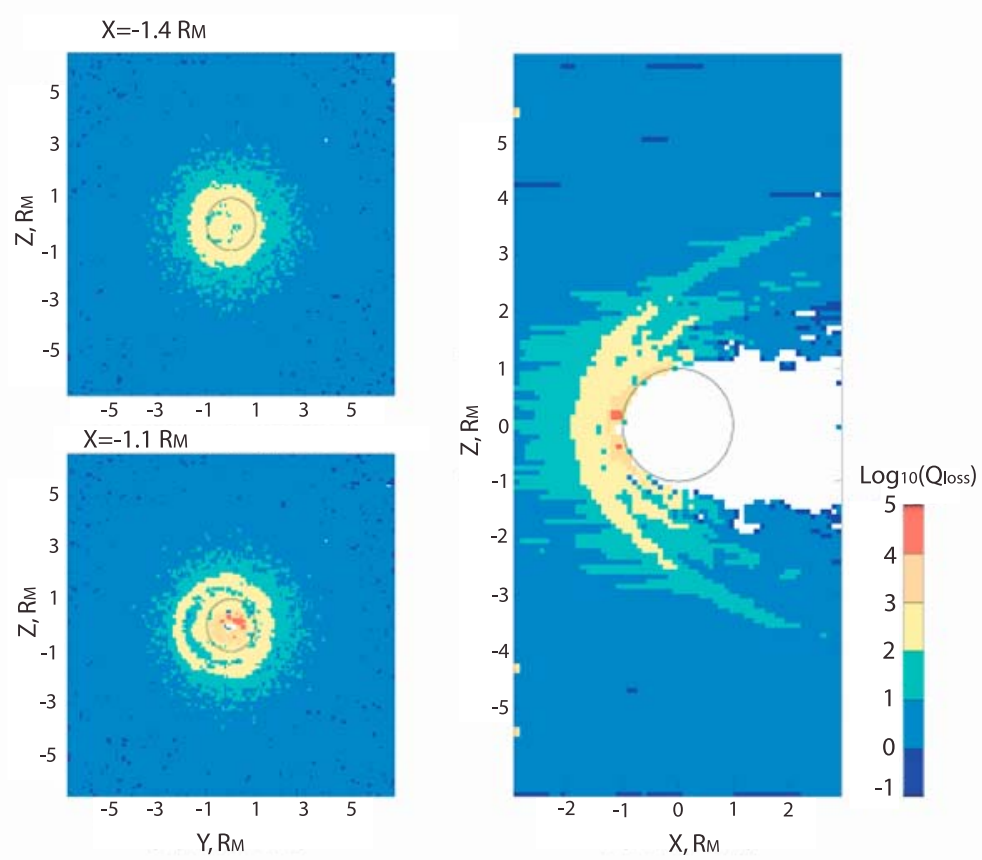

Figure 1. Maps of the removal-rate $Q_{\text {removal }}\left(\mathrm{m}^{-3} \mathrm{~s}^{-1}\right)$ of solar wind $\alpha$-particles in different planes. Y-Z planes at $X=-1.1 R_{M}$ $-1.1 R_{M}$ and $-1.4 R_{M}$ are located upstream of the planet, respectively just downstream of the bow shock and just above the exobase. Plane XZ contains both the bulk velocity of the solar wind and the IMF.

oxygen corona. At high altitude this fraction decreases to $45 \%$ as the contribution from the molecular hydrogen corona, through channel (5), becomes dominant. The same behavior is found during solar maximum conditions although with a smaller importance of the hydrogen corona. Nevertheless the direct production of $\mathrm{He}$ atoms being about 1000 times larger at $300 \mathrm{~km}$ than at $2000 \mathrm{~km}$ we disregard the contribution from the molecular hydrogen corona. In any case, neglecting this production leads to an underestimation of the production of $\mathrm{He}$ atoms.

[8] Figure 1 shows the maps of the removal rates of $\mathrm{He}^{++}$ ions. The right panel depicts the removal rate in the noonmidnight XZ-plane containing the vectors of the solar wind velocity $(+X$ direction $)$ and of the interplanetary magnetic field ( $+Z$ direction). An enhancement of the removal-rate is to be noticed at the location of the bow shock and of its fine structure composed of several "shocklets" in the sheath; it is due to the increase of the density of $\mathrm{He}^{++}$ions. The strongest enhancement of the removal rate is observed near the magnetospheric boundary where the increased residence time of the alpha-particles combines with the increase of the atmospheric number density, leading to an enhanced rate of charge-exchanges. The left plates present maps of the removal-rates in two $\mathrm{YZ}$ cross sections (the motional electric field $-\boldsymbol{V}_{s w} \times \boldsymbol{B}_{I M F}$ is along the Y-axis) at $X=$ $-1.4 R_{M}$ and $X=-1.1 R_{M}$. It is observed that most of removed solar alpha particles have an impact parameter smaller than $\sim 3700 \mathrm{~km}$. The total removal-rate of $\mathrm{He}^{++}$ions is $6.8 \times 10^{23} \mathrm{~s}^{-1}$ which represents $\sim 30 \%$ of the total flux of solar wind alpha-particles through a cross sectional area of radius $r=3700 \mathrm{~km}$, i.e., the planetary radius augmented by the altitude of the exobase. Removed solar wind alpha particles are transformed either into $\mathrm{He}^{+}$ions by single electron transfer on planetary neutrals, or into He atoms by double electron capture. The total removal rate is thus a majorant of the total deposition rate of helium atoms into the Martian atmosphere.

[9] Singly-ionized helium ions originating from the solar wind $\alpha$-particles can be further transformed into neutral atoms by subsequent single electron transfer on Martian neutrals. However the efficiency of two-step processes to produce neutral helium is less than the efficiency of the one-step process by double-electronic capture $\mathrm{He}^{++}+\mathrm{O} \rightarrow \mathrm{He}+\mathrm{O}^{++}$. Part of the produced $\mathrm{He}$ ENAs will impact the exobase and be captured in the lower atmosphere, while another part will escape to space. In general, Monte-Carlo simulations are required to predict the fate of energetic neutrals impacting the atmosphere. Here we present only a simple estimate of the fraction of helium ENAs impacting the exobase. On the entry face of the simulation domain, through which $\mathrm{He}^{++}$ions are injected, we consider four circular and concentric zones having equal areas. The bounding radii of these zones are correspondingly $R_{e x}, \sqrt{2} R_{e x}, \sqrt{3} R_{e x}, 2 R_{e x}$, where $R_{e x}=R_{M}+h_{e x}$ (here $R_{M}=3393 \mathrm{~km}$ is the planetary radius, and $h_{e x}=250 \mathrm{~km}$ is the exobase height). $25000 \mathrm{He}++$ particles/per zone are injected with the same initial statistical weight. The numbers of He-atoms impacting the exobase, and that were produced by 'mother-ions' launched in the different zones, are calculated. The total contributions to the flux of neutral helium from zones $1-4$ are respectively $1.5 \times 10^{23}, 4.4 \times$ $10^{19}, 1.4 \times 10^{18}, 2.6 \times 10^{15}$ atoms s${ }^{-1}$, i.e., $\alpha$-particles 


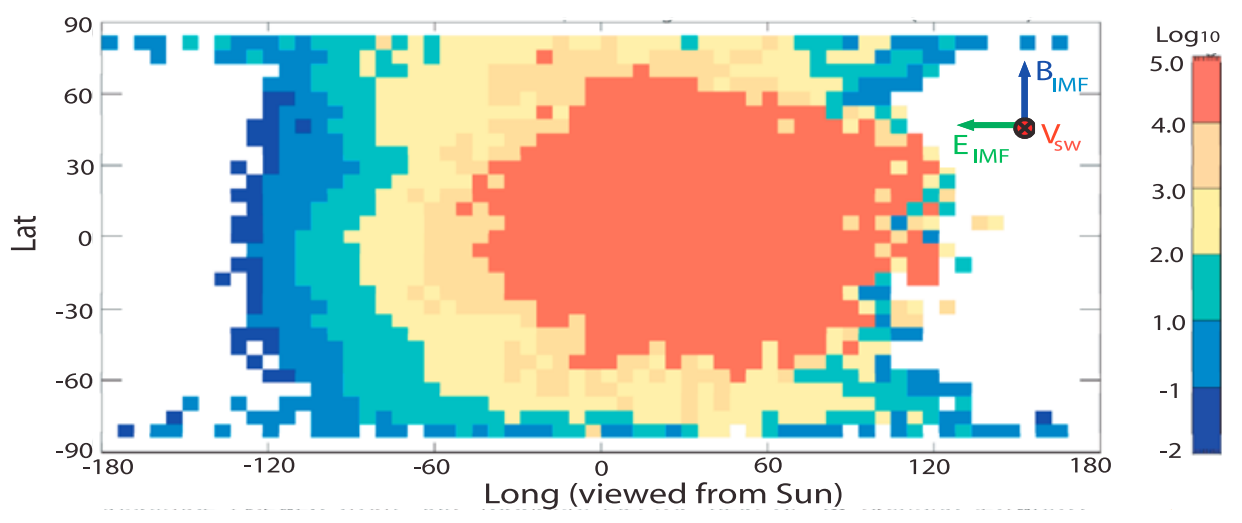

Figure 2. Flux of $\mathrm{He}$-atoms $\left(\mathrm{cm}^{-2} \mathrm{~s}^{-1}\right)$ impacting the exobase of Mars in the latitude-longitude coordinates: latitude and longitude are just names given to the polar angles, they do not have their usual geographical meaning. The point with Lat $=$ 0, Long $=0$ corresponds to the subsolar point. The IMF is directed from the south to north. The interplanetary electric field is antiparallel to the longitude axis.

originating in zone 1 contribute for the major part. Figure 2 shows the latitude-longitude distribution of He-atoms, impacting the exobase, that were produced by 'motherions' injected into the computational domain through zone 1 $(\mathrm{R}=\mathrm{Rex})$. Angles called "latitude" and "longitude" do not have here their usual geographical meaning as long as neither the crustal magnetic field nor the planetary rotation are taken into account: there are indeed just polar angles used to locate a point on a sphere. The interplanetary magnetic and electric fields are parallel to the latitude axis and antiparallel to the longitude axis, respectively. A longitude-asymmetry of the impacts in the $-\boldsymbol{E}_{I M F}$ direction is clearly observed. A similar asymmetry may also be expected in the distribution of neutral helium near Mars, and in the distribution of the sputtered atoms which contribute to the loss processes.

\section{Conclusion}

[10] It has been shown that the capture of solar wind $\alpha$ particles in the exosphere/atmosphere can strongly influence the $\mathrm{He}$ budget on Mars. The removal rate of $\mathrm{He}^{++}$solar wind ions due to charge-exchange processes provides us with an upper limit of the deposition rate of helium on Mars equal to $6.8 \times 10^{23} \mathrm{~s}^{-1}$. This value has the right order of magnitude to compensate for the loss rate of the planetary helium $\left(8 \times 10^{23} \mathrm{~s}^{-1}\right)$ estimated by Krasnopolsky and Gladstone [2005]. $\mathrm{He}^{+}$-ions created by charge exchange can also contribute significantly to the observed fluxes of singly ionized $\mathrm{He}$ at Mars, beside the ionization of neutral helium, and therefore the problem of loss and source processes of helium should be considered self-consistently. Further studies involving Monte-Carlo simulations are needed to refine the helium budget at Mars.

[11] Acknowledgments. E. D. and M. F. wish to acknowledge DLR and DFG for supporting this work by grants FKZ 50 QM 0801 and MO539/ 17-1, respectively. G. C. and R. M. thank MPS (Lindau, Germany) for support of their visits to MPS where this work was started and completed. E. D. acknowledges UVSQ for its support and hospitality during his visits to CETP-IPSL, Velizy. G. C. and R. M. also wish to acknowledge CNES for the financial support of this study.

\section{References}

Acuña, M. H., et al. (1998), Magnetic field and plasma observations at Mars: Initial results of the Mars Global Surveyor MAG/ER experiment, Science, 279, 1676-1680.

Barabash, S., and O. Norberg (1994), Indirect detection of the Martian helium corona, Geophys. Res. Lett., 21, 1547-1550.

Barabash, S., E. Kallio, R. Lundin, and H. Koskinen (1995), Measurements of the nonthermal helium escape from Mars, J. Geophys. Res., 100, $21,307-21,316$.

Barnett, C. F. (Ed.) (1990), Atomic Data for Fusion, vol. 1, Collisions of H, H2, He, Li Atoms and Ions with Atoms and Molecules, Controlled Fusion At. Data Cent., Oak Ridge Natl. Lab., Oak Ridge, Tenn.

Bertucci, C., et al. (2003), Magnetic field draping enhancement at the Martian magnetic pileup boundary from Mars global surveyor observations, Geophys. Res. Lett., 30(2), 1099, doi:10.1029/2002GL015713.

Brecht, S. H. (1997), Solar wind proton deposition into the Martian atmosphere, J. Geophys. Res., 102, 11,287-11,294.

Dubinin, E., et al. (2006), Plasma morphology at Mars. ASPERA-3 observations, Space Sci. Rev., 126, 209-238, doi:10.1007/s11214-006-9039-4.

Haviland, J. K. (1965), The solution of two molecular flow problems by the Monte Carlo method, in Applications in Hydrodynamics, Methods Comput. Phys., vol. 4, edited by B. Alder, S. Fernbach, and M. Rotenberg, pp. 109-209, Academic, New York.

Kim, J., A. F. Nagy, J. L. Fox, and T. E. Cravens (1998), Solar cycle variability of hot oxygen atoms at Mars, J. Geophys. Res., 103, 29,339-29,342.

Kimura, M. (1988), Single- and double-electron capture in $\mathrm{He}^{2+}+\mathrm{He}$ collisions and single-electron capture in $\mathrm{He}^{+}+\mathrm{He}^{+}$collisions, J. Phys. B, 21, 119-124.

Krasnopolsky, V. A. (2002), Mars' upper atmosphere and ionosphere at low, medium, and high solar activities: Implications for evolution of water, J. Geophys. Res., 107(E12), 5128, doi:10.1029/2001JE001809.

Krasnopolsky, V. A., and G. R. Gladstone (1996), Helium on Mars: EUVE and PHOBOS data and implications for Mars' evolution, J. Geophys. Res., 101, 15,765-15,772.

Krasnopolsky, V. A., and G. R. Gladstone (2005), Helium on Mars and Venus: EUVE observations and modeling, Icarus, 176, 395-407.

Krasnopolsky, V. A., S. Bowyer, S. Chakrabarti, G. R. Gladstone, and J. S. McDonald (1994), First measurement of helium on Mars: Implications for the problem of radiogenic gases on the terrestrial planets, Icarus, 109, $337-351$.

Kusakabe, T., H. Yoneda, Y. Mizumoto, and K. Katsurayama (1990), Charge transfer cross sections of ${ }^{3} \mathrm{He}^{2+}$ ions in collisions with $\mathrm{He}$ atoms and $\mathrm{H}_{2}$ molecules in the energy range of $1 \sim 10 \mathrm{keV}$, J. Phys. Soc. Jpn., $59,1218-1224$.

Modolo, R., G. Chanteur, E. Dubinin, and A. Matthews (2005), Influence of the solar activity on the Martian plasma environment, Ann. Geophys., $23,433-444$.

Neukum, G., et al. (2004), Recent and episodic volcanic and glacial activity on Mars revealed by the High Resolution Stereo Camera, Nature, 432, 971-979.

Panov, M. N., V. V. Afrosimov, and A. A. Basalev (2002), Atomic and Plasma Material Interaction Data for Fusion, vol. 10, Int. At. Energy Agency, Vienna.

Vignes, D., C. Mazelle, H. Rme, M. H. Acuña, J. E. P. Connerney, R. P. Lin, D. L. Mitchell, P. Cloutier, D. H. Crider, and N. F. Ness (2000), The solar 
wind interaction with Mars: Locations and shapes of the bow shock and the magnetic pile-up boundary from the observations of the MAG/ER Experiment onboard Mars Global Surveyor, Geophys. Res. Lett., 27, 49-52.

G. M. Chanteur, Laboratoire de Physique des Plasmas, Ecole Polytechnique, Route de Saclay, F-91128 Palaiseau CEDEX, France. (gerard.chanteur@lpp.polytechnique.fr)
E. Dubinin and M. Fraenz, MPS Institut, Max Planck Strasse 2, D-37191 Katlenburg-Lindau, Germany.

R. Modolo, Laboratoire Atmosphères, Milieux, Observations Spatiales, 10 avenue de l'Europe, F-78140 Vélizy CEDEX, France. 